\title{
CRITERIOS PARA LA VALORACIÓN HIDROGEOMORFOLÓGICA DE CURSOS FLUVIALES. APLICACIÓN EN ARAGÓN*
}

\author{
Daniel Ballarín Ferrer, Daniel Mora Mur, Elena Díaz Bea, \\ $\mathbf{M}^{\mathrm{a}}$ Teresa Echeverría Arnedo, Askoa Ibisate González de Matauco, \\ Raquel Montorio Llovería, Alfredo Ollero Ojeda \& Miguel Sánchez Fabre \\ Dpto. de Geografía y Ordenación del Territorio, Facultad de Filosofía y Letras, \\ Universidad de Zaragoza. Pedro Cerbuna, 12, 50009 Zaragoza \\ aollero@unizar.es
}

\begin{abstract}
Resumen: Se ha diseñado un sistema de valoración de indicadores hidrogeomorfológicos de cara a la definición del estado ecológico de los ríos de Aragón. Se emplean distintos indicadores de naturalidad, continuidad y complejidad en la cuenca, el caudal, el cauce y el corredor ribereño. Dividida la red fluvial principal de Aragón en 723 sectores funcionales internamente homogéneos, se ha valorado cada uno de ellos. Los resultados obtenidos son los siguientes: 1.156,34 $\mathrm{km}(17,7 \%$ de la longitud total) en muy buen estado ecológico; $1.697,92 \mathrm{~km}$ (26\%) en buen estado; $1.510,12 \mathrm{~km}(23,1 \%)$ en estado aceptable; $1.221,14 \mathrm{~km}$ $(18,7 \%)$ en estado no aceptable y, finalmente, $939,27 \mathrm{~km}(14,4 \%)$ de la red fluvial muy modificada.
\end{abstract}

Palabras clave: sistemas fluviales, geomorfología fluvial, Directiva 2000/60/CE, estado ecológico, Aragón

\begin{abstract}
A new assessment system based on hydrogeomorphological parameters to determinate the ecological status of rivers in Aragon has been designed. Different indicators have been used: naturalness, continuity and complexity of the basin, discharge, channel and riparian corridor. The fluvial network has been divided in 723 fluvial reaches, functionally homogeneous, which have been evaluated. The results obtained are the following: $1.156,34 \mathrm{~km}(17,7 \%$ of total length) in very good ecological status; $1.697,92 \mathrm{~km}(26 \%)$ in good status; $1.510,12 \mathrm{~km}(23,1 \%)$ in acceptable status; $1.221,14 \mathrm{~km}(18,7 \%)$ in non acceptable status and, finally, $939,27 \mathrm{~km}(14,4 \%)$ of the fluvial network are very modified.
\end{abstract}

* Recibido: 1-12-2005. Aceptado: 31-3-2006. 
Key words: fluvial hydrosystems, fluvial geomorphology, water Framework Directive, ecological status, Aragón

\section{Introducción}

La valoración de un determinado sector funcional de un sistema fluvial puede abordarse desde diferentes puntos de vista, sobre todo si el objetivo es diseñar un sistema de valoración que pueda ser útil para la ordenación del territorio, es decir, para fines como la conservación de los corredores ribereños, la gestión sostenible del agua o la zonificación en función del grado de riesgo, todo ello en un espacio tan complejo y diverso como el fluvial, eje plurifuncional en el que confluyen múltiples intereses.

Casi todos los métodos valorativos de sistemas naturales atienden bien a la valoración intrínseca o per se de los elementos considerados, bien a la valoración de su estado de conservación (si se quiere, de su grado de antropización o de alejamiento del estado natural potencial), o bien a ambos principios. Esta última posibilidad es más completa e integradora y más adecuada para la ordenación o gestión del sistema estudiado y evaluado. Es decir, la valoración final sería el resultado de la combinación del valor intrínseco y del valor o estado conservacional. Pero, además, el método debería permitir la exposición de los resultados parciales de cada una de las dos valoraciones, ya que para determinados objetivos puede interesar únicamente una de ellas.

Sin embargo, en aplicación de la Directiva 2000/60/CE, el objetivo es valorar exclusivamente el estado ecológico de conservación actual de los ríos europeos. Entre las recomendaciones publicadas hasta el momento destacan las del documento de la Commission of the European Communities (2002) Guidance standard CEN TC 230/WG 2/TG 5: N32 y las del grupo de trabajo 2.3 REFCOND (Wallin et al., 2002). El equipo de trabajo, tras numerosas discusiones metodológicas y el análisis de diferentes versiones del sistema de valoración, tuvo que centrarse, por tanto, en la evaluación del grado de naturalidad/antropización hidromorfológica, en el marco de un reconocimiento inicial del estado ecológico de los ríos de Aragón. Así, la versión que se presenta en este artículo está enfocada hacia el resultado de los impactos o procesos de antropización en el funcionamiento del sistema fluvial y en concreto sobre la cuenca, sobre el caudal, sobre el cauce y sobre el corredor ribereño.

En muchos países han aparecido en la última década planes de ordenación, programas de conservación y restauración y normativas diversas que afectan a cauces y 
riberas. Algunas de ellas cuentan con îndices de valoración hidromorfológica o, más habitualmente, con índices ecológicos que valoran también algunos parámetros hidromorfológicos. Pueden destacarse en el Reino Unido el "River Habitat Survey" (RHS) (Raven et al., 1998), en Francia el "SEQ-Physique" (Système d'Evaluation de la Qualité Physique des Cours d'Eau) (Tartar, 2001), en Alemania el Länderarbeitsgemeinschaft Wasser (LAWA, 2000) o en Italia el "Indice de Funcionalitá Fluviale" (IFF) (Siligardi, coord, 2003). Uno de los índices más interesantes es el "Index of Stream Condition" (ISC) (Ladson \& White, 1999), que se integra en el programa AUSRIVAS (Australian River Assessment System) (Parsons et al., 2002). En España se han desarrollado algunos índices de calidad de espacios ribereños, como el QBR (Qualitat del Bosc de Ribera) (Munné et al., 1998) o el IHF-GUADALMED (Bonada et al., 2002).

\section{Metodología básica}

Esta evaluación inicial del estado ecológico de los ríos de Aragón desde una perspectiva hidromorfológica se ha apoyado en tres fuentes de información: la obtenida de trabajos preexistentes, bases de datos y la experiencia personal de los miembros del equipo de estudio, la procedente de la cartografía básica de los sistemas fluviales y de la consulta de las fotografías aéreas más recientes y la emanada del trabajo de campo desarrollado sobre los puntos de muestreo.

Así pues, la detallada caracterización de la red fluvial aragonesa llevada a cabo, diferenciando distintos tipos de sectores fluviales y catalogando en ellos cada tramo de la red fluvial, ha sido fundamental para la valoración de los indicadores. No toda la información recopilada desde la caracterización ha sido necesaria para la valoración inicial del estado ecológico, sino que se ha llevado a cabo un proceso de selección de indicadores hidromorfológicos para ser éstos los que, en suma, han sido valorados. En los sectores funcionales de la red fluvial en los que no se ha podido realizar trabajo de campo en punto de muestreo el equipo de trabajo ha estimado una valoración general, apoyada en la información disponible y asentada también en la comparación con otros sectores funcionales del mismo curso fluvial en los que sí se ha podido abordar trabajo de campo y, por tanto, valoración exacta.

La mayor parte del trabajo se realizó en la segunda mitad de los años 2002 y 2003, es decir, entre los meses de julio y diciembre, aunque se llevaron a cabo salidas puntuales también en 2004. 


\section{Definición de indicadores hidromorfológicos}

Para este paso imprescindible de selección y definición de indicadores se han atendido, consultado o tenido en cuenta los cuatro siguientes documentos, criterios o premisas (Ollero et al., 2004):

a) Los indicadores hidromorfológicos que afectan a los biológicos del apartado 1.1.1 del Anexo V de la Directiva, según definiciones de los apartados 1.2 y 1.2.1 del anexo $\mathrm{V}$ :

-Régimen hidrológico:

Caudales e hidrodinámica del flujo de las aguas

Conexión con masas de agua subterránea

-Continuidad del río

-Condiciones morfológicas:

Variación de la profundidad y anchura del río

Estructura y sustrato del lecho del río

Estructura de la zona ribereña

b) El Guidance standard CEN TC 230/WG 2/TG 5: N32, que recomienda que la selección de indicadores y el establecimiento de condiciones de referencia se elabore atendiendo a la estructura transversal de los sistemas fluviales en tres elementos: a) cauce, b) orillas y corredor ribereño, c) llano de inundación. Este documento también establece cinco conjuntos de variables que considera muy interesantes como referencia a seguir en el proceso de trabajo: a) características del lecho y orillas, b) planta y perfil del río, c) conectividad y libertad de movimiento lateral, d) flujo libre del agua y de los sedimentos en el canal y e) vegetación de ribera.

c) El hecho de que el estado ecológico puede cambiar con el paso del tiempo, de manera que algunos indicadores deberían ser válidos para medir esta evolución. Por ejemplo, seria adecuado el rate of bydromorphological change (Commission of the European Communities, 2002), que valora el número y carácter de variaciones en el sistema a lo largo de un periodo fijo ( 5 ó 10 años). Sin embargo, por las condiciones del trabajo realizado y su carácter pionero, la dinámica temporal sólo habría podido ser evaluada en todo caso con carácter retrospectivo (mediante fotos aéreas antiguas o datos de caudal). Sí que será muy interesante, por ello, establecer y medir sobre el terreno indicadores prospectivos, que puedan volver a evaluarse en futuros estudios.

d) La experiencia previa del equipo de trabajo en el análisis y valoración de sistemas fluviales, de acuerdo con la cual es preciso considerar un conjunto de caracte- 
res o valores interrelacionados que definen o aportan una valoración alta del estado ecológico en un sistema fluvial:

-Naturalidad en el comportamiento hidrológico, geomorfológico y ecológico del sistema, como contraposición a la antropización del mismo.

-Continuidad, valor relacionado con el carácter unitario del sistema fluvial y de su cuenca vertiente. La correcta continuidad del curso fluvial, es decir, la ausencia de barreras en el mismo, es clave para garantizar su papel como corredor ecológico.

-Complejidad, tanto longitudinal (sucesión de tramos internamente homogéneos pero diferentes entre sî) como transversal (ambientes del cauce y las riberas), garantía de biodiversidad, por el carácter de ecotono y la presencia de varias interfases con gran cantidad de interrelaciones e interacciones.

-Funcionalidad del sistema fluvial, correcto funcionamiento como mecanismo de movilización o conducción superficial de las aguas acompañadas de los materiales que transportan, sedimentos y nutrientes, en la dirección de la pendiente.

-Dinámica espacio-temporal del sistema fluvial, de una intensidad generalmente alta, poco frecuente en la naturaleza, conformada por complejos procesos activos y metamorfosis tanto en el eje longitudinal como en el transversal como en la vertical. La dinámica garantiza la conectividad longitudinal (entre tramos) y transversal (entre bandas) asegurando la conservación de las interrelaciones, de los ecosistemas y del continuo fluvial.

-Singularidad, valor relacionado con la representatividad y la calidad paisajística.

A partir de todas las anteriores premisas se han seleccionado los indicadores concretos que, desde una perspectiva hidromorfológica, han servido para especificar las condiciones de referencia, para informar del estado de cada tramo de río y, en suma, para contribuir a determinar el estado ecológico de toda la red.

\subsection{Indicadores de naturalidad}

Son numerosos y relevantes, desde el momento en que el principal objetivo de la valoración en el presente estudio es el estado ecológico, y no el valor intrínseco

a) Naturalidad de la cuenca: se evalúa el grado de antropización de la cuenca vertiente al sector funcional, observando en cartografía, fotografía aérea y otras fuen- 
tes (censos, publicaciones...) los usos del suelo, las actividades humanas y la población residente de forma permanente o discontinua. La complejidad de los datos obliga a una valoración cualitativa simplificada ( 4 muy alta, 3 alta, 2 media, 1 baja) que necesariamente se basa en la comparación de unas cuencas con otras.

b) Naturalidad del volumen de caudal hídrico: se evalúa, trabajando como mínimo sobre valores mensuales, en qué medida los volúmenes de caudal hídrico circulantes por el sistema fluvial en el sector funcional corresponden con los naturales o bien son reducidos (detracciones y derivaciones para diferentes usos humanos, embalses, hidroeléctricas, riegos, abastecimiento urbano o industrial, etc.) o incrementados (aportes artificiales desde otras cuencas). La aplicación de puntos es la siguiente: 4 caudal natural, 3 detracciones inferiores al 10\% del caudal natural, 2 detracciones entre el 10 y el 20\%, 1 detracciones entre el 20 y el 50\%, 0 detracciones superiores al $50 \%$.

c) Naturalidad del volumen de caudal sólido: se evalúa en qué medida los volúmenes de caudal sólido circulantes por el sistema fluvial en el sector funcional corresponden con los naturales o bien son reducidos por retención de sedimentos en presas. La aplicación de puntos es la siguiente: 4 caudal natural, 3 con menos del 20\% de la superficie de drenaje de la cuenca afectado por presas, 2 del 20 al 50\% de la superficie de la cuenca afectado por presas, 1 del 50 al $90 \%$ de la cuenca afectado por presas, 0 más del $90 \%$ de la cuenca afectado por presas.

d) Naturalidad del régimen hidrológico: se comprueba si está alterado o no el régimen natural estacional por infraestructuras con capacidad de regulación o de derivación de caudales aguas arriba. En este caso se aplican tres posibilidades: 4 régimen natural, 2 régimen algo modificado, 0 régimen modificado. La intermedia se establece cuando hay modificaciones pero no son significativas, alterando en poca medida la curva natural de caudales a lo largo de los meses del año, de acuerdo con la estimación o el estudio de datos realizado por los evaluadores.

e) Naturalidad longitudinal del cauce: se evalúa el grado de antropización a partir de la detección de impactos longitudinales (rupturas de pendiente de origen antrópico, dragados, canalizaciones, rectificaciones del cauce) en foto aérea y campo. La escala aplicable es 5 inalterada, 4 mínimamente alterada, 3 algo alterada, 2 bastante alterada, 1 muy alterada.

f) Naturalidad de la sección transversal del cauce: igualmente se evalúa el grado de antropización a partir de la detección de impactos transversales (defensas, dragados, vados, caminos, extracciones de áridos, etc.) en foto aérea y campo. La 
escala aplicable es 5 inalterada, 4 mínimamente alterada, 3 algo alterada, 2 bastante alterada, 1 muy alterada.

g) Naturalidad de la componente vertical del sistema y de la conexión con el freático: se valora a partir de observaciones de campo el estado del sustrato del lecho y del freático, detectando impactos que lo alteran, como embalses, pozos, dragados, defensas, incisiones de drenaje, etc. La puntuación aplicada es: 5 inalterada, 4 mínimamente alterada, 3 algo alterada, 2 bastante alterada, 1 muy alterada.

h) Naturalidad de la conexión del cauce con las vertientes: se observa en campo y foto aérea la presencia o ausencia de infraestructuras (defensas, vías de comunicación...) o procesos antrópicos (movimientos de tierras...) que pueden romper dicha conexión, separando drásticamente los dos ámbitos. Esa separación implica la dificultad o imposibilidad de la llegada de sedimentos desde los procesos de vertiente para ser evacuados por la red fluvial. La puntuación aplicada es también: 5 inalterada, 4 mínimamente alterada, 3 algo alterada, 2 bastante alterada, 1 muy alterada.

i) Anchura o desarrollo transversal del corredor ribereño: es indicador clave de su naturalidad, ya que en la mayor parte de los casos las riberas han sido constreñidas por invasiones humanas, puesta en cultivo, talas, urbanización, defensas... La valoración es también: 5 inalterada, 4 mínimamente alterada, 3 algo alterada, 2 bastante alterada, 1 muy alterada. Sólo se aplica este indicador a cursos con ribera.

j) Naturalidad de especies de ribera: valor asociado en la puntuación a la diversidad, trata de estimar el grado de antropización de las riberas a partir de la detección de impactos y de la observación cualitativa en campo del estado de conservación de la vegetación de ribera natural. Sólo se aplica este indicador a cursos con ribera. La puntuación asignada es la siguiente: 4 alta, 3 media, 2 baja, 1 muy baja, siendo fundamental la experiencia de los evaluadores para su determinación.

k) Naturalidad de la conexión del corredor ribereño con las vertientes: como en el caso de la conexión cauce-vertiente, se observa en campo y foto aérea la presencia o ausencia de infraestructuras (en especial vías de comunicación) que pueden romper dicha conexión, separando drásticamente corredor ribereño y vertiente y, con ello, los procesos geomorfológicos y de interconexión vegetal. La puntuación aplicada es en ese caso: 4 inalterada, 3 algo alterada, 2 bastante alterada, 1 muy alterada. Sólo se aplica este indicador a cursos con ribera. 


\subsection{Indicadores de continuidad}

a) Continuidad longitudinal del cauce desde el inicio hasta el final del sector funcional estudiado. Se observa en cartografía, fotografía aérea y campo la presencia o no de infraestructuras transversales al cauce que constituyan una barrera para los flujos hidromorfológicos y ecológicos que circulan en la dirección del drenaje: presas, azudes y, en menor medida, puentes. La puntuación es la siguiente: 5 sin infraestructuras-barrera, $4<0,25$ infraestructuras $/ \mathrm{km}, 3$ de 0,25 a 0,5 infraestructuras $/ \mathrm{km}, 2$ de 0,5 a 0,75 infraestructuras $/ \mathrm{km}, 1$ de 0,75 a 1 infraestructuras $/ \mathrm{km}, 0>1$ infraestructura $/ \mathrm{km},-5$ al menos una presa de más de $5 \mathrm{~m}$ de altura.

b) Continuidad longitudinal del corredor ribereño, sólo evaluada en los cursos no encajados, es decir, en aquellos sistemas que pueden contar con dicho corredor. Se comprueba en fotografías aéreas observando en el conjunto del tramo las rupturas del corredor en cada una de las dos orillas. Las causas de dichas rupturas suelen ser invasiones, puesta en cultivo, talas, urbanización, defensas... La puntuación aplicada es: 3 alta, 2 media, 1 baja, 0 nula.

\subsection{Indicadores de complejidad}

Son cinco, aunque en el presente estudio se han integrado en cuatro parámetros a evaluar. Sólo se aplican a cursos con ribera.

a) Diversidad de estilos fluviales: se valora positivamente la complejidad longitudinal de la geomorfología del cauce y del corredor ribereño y negativamente la excesiva homogeneidad interna del sector funcional. Para evaluar este indicador es preciso un cambio de escala y una observación en fotografía aérea y campo de mayor detalle que la que se utilizó para delimitar los sectores funcionales (Ollero et al., 2003). En efecto, se entiende que cuando se diferenciaron los sectores o tramos se definió cada uno de ellos como "internamente homogéneo" en cuanto a su estilo fluvial. Sin embargo, si se observa el tramo con mayor detalle siempre encontraremos modificaciones locales de estilo (subestilos, si se quiere). Por ejemplo, dentro de un cañón hay subtramos más abiertos, otros con paredes extraplomadas (oscuros), otros con depósitos de gravas que subdividen la corriente, otros de cauce sinuoso, etc. Esa diversidad es la que se evalúa. No obstante, aunque es un parámetro muy importante para la valoración de la calidad intrínseca, no lo es tanto para la valoración del estado ecológico, objetivo del presente estudio, salvo que se deba a impactos antrópicos la homogeneización o pérdida de diversidad. Por ello, se le ha restado importancia integrándolo en la puntuación del siguiente parámetro (diversidad de ambientes). 
b) Diversidad de ambientes: se contabiliza el número de ambientes o biotopos diferentes (por ejemplo: el lecho inundado, una barra de gravas, un depósito de finos, un meandro abandonado...) que se encuentran a lo largo del perfil transversal representativo del cauce y sus riberas llevado a cabo en el punto de muestreo. Los evaluadores otorgan la puntuación final valorando tanto esta diversidad transversal de ambientes como la longitudinal de estilos fluviales (indicador anterior), atendiendo en ambos casos a si las reducciones de diversidad se deben a factores naturales o antrópicos (defensas, dragados, relleno de cauces secundarios o abandonados...). Sólo en el segundo de los casos se penalizan en la puntuación, cuya escala es la siguiente: 5 inalterada, 4 mínimamente reducida, 3 algo reducida, 2 bastante reducida, 1 muy reducida.

c) Interconectividad de ambientes, observada en cada una de las dos orillas en el punto de muestreo, a lo largo de la sección transversal. En principio es correcta si no hay elementos antrópicos (caminos, defensas, edificios) que separen las bandas de ribera o las interrumpan internamente. Conviene completar la observación con fotos aéreas para abarcar en la medida de lo posible todo el tramo. La aplicación de puntos es la siguiente: 3 correcta en las dos orillas, 2 correcta en una orilla y deficiente en la otra, 1 deficiente en las dos orillas. Si la situación de cada una de las orillas es compleja los evaluadores aplican una alternativa evidente: 3 correcta en líneas generales, 2 situación intermedia o con algunas deficiencias, 1 globalmente deficiente.

d) Estructura de las riberas: se comprueba el grado de conservación de la estructura en bandas del corredor ribereño tal como se observa en la zona del punto de muestreo, confirmándola en fotografía aérea. Normalmente es alterada por pastoreo, talas, usos recreativos, extracciones, invasiones, introducción de especies exóticas, repoblaciones, etc. La puntuación aplicada es: 5 inalterada, 4 mínimamente alterada, 3 algo alterada, 2 bastante alterada, 1 muy alterada.

e) Diversidad de especies de ribera, que se evalúa a partir del recuento de especies exclusivas de riberas fluviales que se lleva a cabo en cada punto de muestreo. Este indicador se aplica de forma conjunta con la naturalidad de especies de ribera (más arriba en 3.1.j), aplicando la puntuación: 4 alta, 3 media, 2 baja, 1 muy baja. 


\section{Sistema de valoración}

Para cada sector funcional en el que se ha desarrollado trabajo de campo en puntos de muestreo se ha podido llevar a cabo la valoración hidromorfológica, empleándose una ficha (figura 1) en la que se recogen 16 indicadores de los explicados en el apartado anterior.

\begin{tabular}{|c|c|c|c|c|c|c|c|}
\hline \multicolumn{8}{|c|}{$\begin{array}{l}\text { FICHA DE VALORACIÓN DEL ESTADO ECOLÓGICO DEL SECTOR } \\
\text { FUNCIONAL A PARTIR DE INDICADORES HIDROMORFOLÓGICOS }\end{array}$} \\
\hline \multicolumn{2}{|c|}{ sistema fluvial } & sector func. & tipo & & & fecha & \\
\hline \multicolumn{4}{|c|}{ caracteres indicadores y sistema de valoración } & $\begin{array}{l}\text { ponde- } \\
\text { ración }\end{array}$ & $\begin{array}{c}\text { valor } \\
\text { en el } \\
\text { sector }\end{array}$ & $\begin{array}{c}\% \\
\text { resp. } \\
\text { máximo }\end{array}$ & $\begin{array}{l}\text { causas } \\
\text { de alteración }\end{array}$ \\
\hline \multicolumn{4}{|c|}{$\begin{array}{l}\text { naturalidad de la cuenca } \\
4 \text { muy alta, } 3 \text { alta, } 2 \text { media, } 1 \text { baja }\end{array}$} & $\mathbf{x} 1$ & & & \\
\hline \multirow{3}{*}{ 它 } & \multicolumn{3}{|c|}{$\begin{array}{l}\text { naturalidad del volumen de caudal hidrico } \\
4 \text { caudal natural, } 3 \text { detracciones inferiores al } 10 \% \text { del } \\
\text { caudal natural, } 2 \text { detracciones entre el } 10 \text { y el } 20 \% \text {, } \\
1 \text { detracciones entre el } 20 \text { y el } 50 \%, 0 \text { detracciones } \\
\text { superiores al } 50 \%\end{array}$} & $\times \mathbf{x} 2$ & & & \\
\hline & \multicolumn{3}{|c|}{$\begin{array}{l}\text { naturalidad del volumen de caudal sólido } \\
4 \text { caudal natural, } 3 \text { con menos del } 20 \% \text { de la cuenca } \\
\text { afectado por presas, } 2 \text { del } 20 \text { al } 50 \% \text { de la cuenca afec- } \\
\text { tado por presas, } 1 \text { del } 50 \text { al } 90 \% \text { de la cuenca afectado } \\
\text { por presas, } 0 \text { más del } 90 \% \text { de la cuenca afectado por } \\
\text { presas }\end{array}$} & $\mathbf{x} 2$ & & & \\
\hline & \multicolumn{3}{|c|}{$\begin{array}{l}\text { naturalidad del régimen hidrológico } \\
4 \text { régimen natural, } 2 \text { régimen algo modificao, } 0 \text { régimen } \\
\text { modificado }\end{array}$} & $\mathbf{x} 2$ & & & \\
\hline \multirow{5}{*}{ 幽 } & \multicolumn{3}{|c|}{$\begin{array}{l}\text { continuidad longitudinal } \\
5 \text { sin infraestructuras-barrera, } 4<0,25 \text { infraestructuras } / \mathrm{km} \text {, } \\
3 \text { de } 0,25 \text { a } 0,5 \text { infraestructuras } / \mathrm{km}, 2 \text { de } 0,5 \text { a } 0,75 \\
\text { infraestructuras } / \mathrm{km}, 1 \text { de } 0,75 \text { a } 1 \text { infraestructuras } / \mathrm{km}, 0> \\
1 \text { infraestructuras } / \mathrm{km},-5 \text { al menos una presa de más de } \\
5 \mathrm{~m} \text { de altura }\end{array}$} & $\mathbf{x} 2$ & & & \\
\hline & \multicolumn{3}{|c|}{$\begin{array}{l}\text { naturalidad longitudinal } \\
5 \text { inalterada, } 4 \text { mínimamente alterada, } 3 \text { algo alterada, } \\
2 \text { bastante alterada, } 1 \text { muy alterada }\end{array}$} & $\mathbf{x} 2$ & & & \\
\hline & \multicolumn{3}{|c|}{$\begin{array}{l}\text { naturalidad de la sección transversal } \\
5 \text { inalterada, } 4 \text { mínimamente alterada, } 3 \text { algo alterada, } \\
2 \text { bastante alterada, } 1 \text { muy alterada }\end{array}$} & $\mathbf{x} 2$ & & & \\
\hline & \multicolumn{3}{|c|}{$\begin{array}{l}\text { naturalidad de la componente vertical del sistema y } \\
\text { de la conexión con el freático } \\
5 \text { inalterada, } 4 \text { mínimamente alterada, } 3 \text { algo alterada, } \\
2 \text { bastante alterada, } 1 \text { muy alterada }\end{array}$} & $\mathbf{x} \mathbf{1}$ & & & \\
\hline & \multicolumn{3}{|c|}{$\begin{array}{l}\text { naturalidad de la conexión con las vertientes } \\
5 \text { inalterada, } 4 \text { mínimamente alterada, } 3 \text { algo alterada, } \\
2 \text { bastante alterada, } 1 \text { muy alterada }\end{array}$} & $\mathbf{x} 1$ & & & \\
\hline
\end{tabular}




\begin{tabular}{|c|c|c|c|c|c|c|c|}
\hline \multicolumn{8}{|c|}{$\begin{array}{l}\text { FICHA DE VALORACIÓN DEL ESTADO ECOLÓGICO DEL SECTOR } \\
\text { FUNCIONAL A PARTIR DE INDICADORES HIDROMORFOLÓGICOS }\end{array}$} \\
\hline \multicolumn{2}{|c|}{ sistema fluvial } & sector func. & tipo & & & fecha & \\
\hline \multicolumn{4}{|c|}{ caracteres indicadores y sistema de valoración } & $\begin{array}{l}\text { ponde- } \\
\text { ración }\end{array}$ & $\begin{array}{c}\text { valor } \\
\text { en el } \\
\text { sector }\end{array}$ & $\begin{array}{c}\% \\
\text { resp. } \\
\text { máximo }\end{array}$ & $\begin{array}{l}\text { causas } \\
\text { de alteraciôn }\end{array}$ \\
\hline \multirow{7}{*}{ 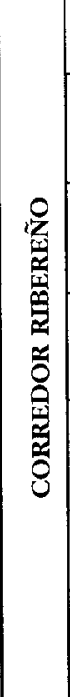 } & \multicolumn{3}{|c|}{$\begin{array}{l}\text { continuidad longitudinal } \\
3 \text { alta, } 2 \text { media, } 1 \text { baja, } 0 \text { nula }\end{array}$} & $\mathbf{x} 2$ & & & \\
\hline & \multicolumn{3}{|c|}{$\begin{array}{l}\text { anchura o desarrollo transversal } \\
5 \text { inalterada, } 4 \text { mínimamente reducida, } 3 \text { algo reducida, } \\
2 \text { bastante reducida, } 1 \text { muy reducida }\end{array}$} & $\mathbf{x} 1$ & & & \\
\hline & \multicolumn{3}{|c|}{$\begin{array}{l}\text { interconectividad de ambientes } \\
5 \text { inalterada, } 4 \text { mínimamente reducida, } 3 \text { algo reducida, } \\
2 \text { bastante reducida, } 1 \text { muy reducida. }\end{array}$} & $\mathbf{x} 1$ & & & \\
\hline & \multicolumn{3}{|c|}{$\begin{array}{l}\text { interconectividad de ambientes } \\
3 \text { correcta en las dos orillas, } 2 \text { correcta en una orilla y } \\
\text { deficiente en la otra, } 1 \text { deficiente en las dos orillas }\end{array}$} & $\mathbf{x 1}$ & & & \\
\hline & \multicolumn{3}{|c|}{$\begin{array}{l}\text { estructura de ambientes } \\
5 \text { inalterada, } 4 \text { mínimamente alterada, } 3 \text { algo alterada, } \\
2 \text { bastante alterada, } 1 \text { muy alterada }\end{array}$} & $\mathbf{x} 1$ & & & \\
\hline & \multicolumn{3}{|c|}{$\begin{array}{l}\text { naturalidad y diversidad de especies de ribera } \\
4 \text { alta, } 3 \text { media, } 2 \text { baja, } 1 \text { muy baja }\end{array}$} & $\mathbf{x} 1$ & & & \\
\hline & \multicolumn{3}{|c|}{$\begin{array}{l}\text { naturalidad de la conexión con las vertientes } \\
4 \text { inalterada, } 3 \text { algo alterada, } 2 \text { bastante alterada, } 1 \text { muy } \\
\text { alterada }\end{array}$} & $\mathbf{x ~} 1$ & & & \\
\hline \multicolumn{5}{|c|}{ VALORACIÓN FINAL } & & & $\begin{array}{l}\text { Nota: el corredor } \\
\text { ribereño sólo se } \\
\text { valora en tipos } \\
\text { con ribera }\end{array}$ \\
\hline
\end{tabular}

Figura 1. Ficha de valoración inicial del estado ecológico a partir de indicadores hidromorfológicos.

Cada indicador cuenta con su escala de valores, recogida en la propia ficha en letra pequeña para su consulta directa facilitando el trabajo de los evaluadores. Algunos indicadores presentan una escala cuantitativa y la mayoría reflejan una respuesta cualitativa que se logra por el consenso de todo el equipo de trabajo.

Cada uno de los 16 indicadores hidromorfológicos asiste a un proceso de ponderación, ya que el equipo de trabajo ha creído conveniente valorar más unos que otros de cara al objetivo final de la definición del estado ecológico. Puede observarse en la ficha que hay siete indicadores valorados al doble. También existe una cierta ponderación en la propia escala de puntuaciones, ya que hay varios indicadores cuya puntuación máxima es 5 , otros 4 y alguno 3. 
En suma, los 16 valores evaluados cuentan con mayor o menor importancia en la valoración final del sector funcional del siguiente modo:

-Valor máximo 10: continuidad longitudinal del cauce, naturalidad longitudinal del cauce, naturalidad de la sección transversal del cauce.

-Valor máximo 8: naturalidad del volumen de caudal hídrico, naturalidad del volumen de caudal sólido, naturalidad del régimen hidrológico.

-Valor máximo 6: continuidad longitudinal del corredor ribereño.

-Valor máximo 5: naturalidad de la componente vertical del sistema y de la conexión del cauce con el freático, naturalidad de la conexión del cauce con las vertientes, desarrollo transversal del corredor ribereño, diversidad de ambientes en el corredor ribereño, estructura de ambientes.

-Valor máximo 4: naturalidad de la cuenca, naturalidad y diversidad de especies de ribera, naturalidad de la conexión del corredor ribereño con las vertientes.

-Valor máximo 3: interconectividad de ambientes en el corredor ribereño.

Así, la cuenca vertiente puede obtener hasta 4 puntos en la valoración, el caudal hasta 24 puntos, el cauce hasta 40 puntos y el corredor ribereño hasta 32 puntos, sumando todo ello 100 puntos. Por lo que se refiere a grandes grupos de valores, la naturalidad supone hasta 64 puntos, la continuidad hasta 16 y la complejidad hasta 20 puntos.

La última fase del proceso de valoración consiste en comparar el resultado (la suma de los 16 valores indicadores una vez ponderados) con el valor máximo posible ( 100 en cursos con ribera, 68 en cursos sin ribera), obteniéndose el porcentaje de desvío respecto a dicho valor máximo. En función de tal porcentaje se ha elaborado una escala que reúne los resultados de la valoración hidromorfológica en 5 grandes grupos, aplicables posteriormente al proceso de combinación con las otras variables estudiadas para alcanzar la valoración final del estado ecológico:

a) Muy buen estado hidromorfológico (color verde oscuro en el mapa, fig. 2): sectores funcionales cuya valoración de indicadores hidromorfológicos sea al menos del $85 \%$ respecto de la valoración máxima, es decir, 85 puntos o más en cursos con ribera y más de 57 puntos en cursos sin ribera

b) Buen estado hidromorfológico (color verde claro en el mapa, fig. 2): sectores funcionales cuya valoración de indicadores hidromorfológicos se encuentre entre el 70 y el $85 \%$ respecto de la valoración máxima, es decir, con puntuaciones entre 70 y 84 en cursos con ribera y entre 48 y 56 en cursos sin ribera. 
c) Estado hidromorfológico aceptable (color amarillo en el mapa, fig. 2): sectores funcionales cuya valoración de indicadores hidromorfológicos se encuentre entre el 55 y el 70\% respecto de la valoración máxima, es decir, con puntuaciones entre 55 y 69 en cursos con ribera y entre 38 y 47 en cursos sin ribera.

d) Estado hidromorfológico no aceptable (color naranja en el mapa, fig. 2): sectores funcionales cuya valoración de indicadores hidromorfológicos se encuentre entre el 40 y el 55\% respecto de la valoración máxima, es decir, con puntuaciones entre 40 y 54 puntos en cursos con ribera y entre 28 y 37 puntos en cursos sin ribera.

e) Estado hidromorfológico muy modificado (color rojo en el mapa, fig. 2): sectores funcionales cuya valoración de indicadores hidromorfológicos es inferior al $40 \%$ respecto de la valoración máxima, es decir, con menos de 40 puntos en cursos con ribera y con menos de 28 puntos en cursos sin ribera.

\section{Resultados y conclusiones}

A partir de los indicadores y el sistema expuestos, se han valorado los 332 sectores funcionales de la red fluvial principal de Aragón en los que se ha llevado a cabo muestreo de campo y, además, se ha estimado la valoración de los 391 sectores restantes ( $\sin$ muestreo de campo). Es imposible incluir en este artículo las 723 fichas de valoración que constituyen la información de base del trabajo realizado. En el mapa de la figura 2 se resumen los resultados obtenidos sobre la red fluvial de Aragón analizada.

Las conclusiones más relevantes que pueden extraerse de los resultados obtenidos son las siguientes:

a) En total hay 192 sectores funcionales (el 26,6\%) cuyo estado hidromorfológico es muy bueno, 220 sectores $(30,4 \%)$ en estado bueno, 142 sectores $(19,6 \%)$ en estado aceptable, 79 sectores $(10,9 \%)$ en estado no aceptable y 90 sectores $(12,4 \%)$ en estado muy modificado (figura 3). Si tenemos en cuenta la longitud de la red fluvial (figura 4), los sectores funcionales en muy buen estado hidromorfológico suman 1.156,34 $\mathrm{km}$, por lo que su peso relativo (el 17,7\%) es menor que el que indicaba su número. Los sectores en estado bueno alcanzan $1.697,92 \mathrm{~km}$, es decir, el $26 \%$ de la longitud total de la red fluvial estudiada. Los sectores en estado aceptable suman $1.510,12 \mathrm{~km}$ $(23,1 \%)$, los sectores en estado no aceptable alcanzan $1.221,14 \mathrm{~km}(18,7 \%)$ y los sectores muy modificados $939,27 \mathrm{~km}(14,4 \%)$. 


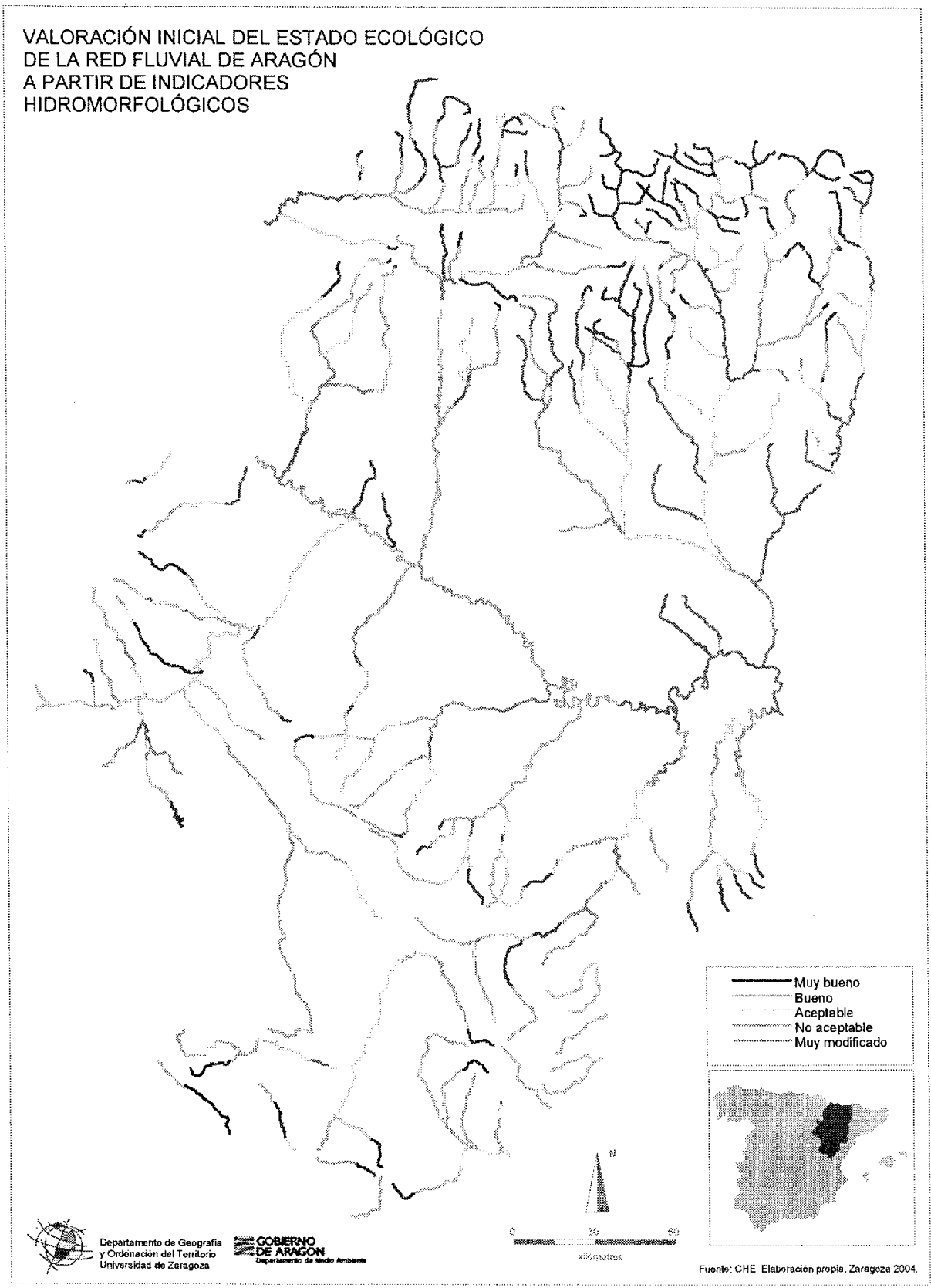

Figura 2. Mapa del estado ecológico de los ríos de Aragón en función de parámetros hidromorfológicos. 


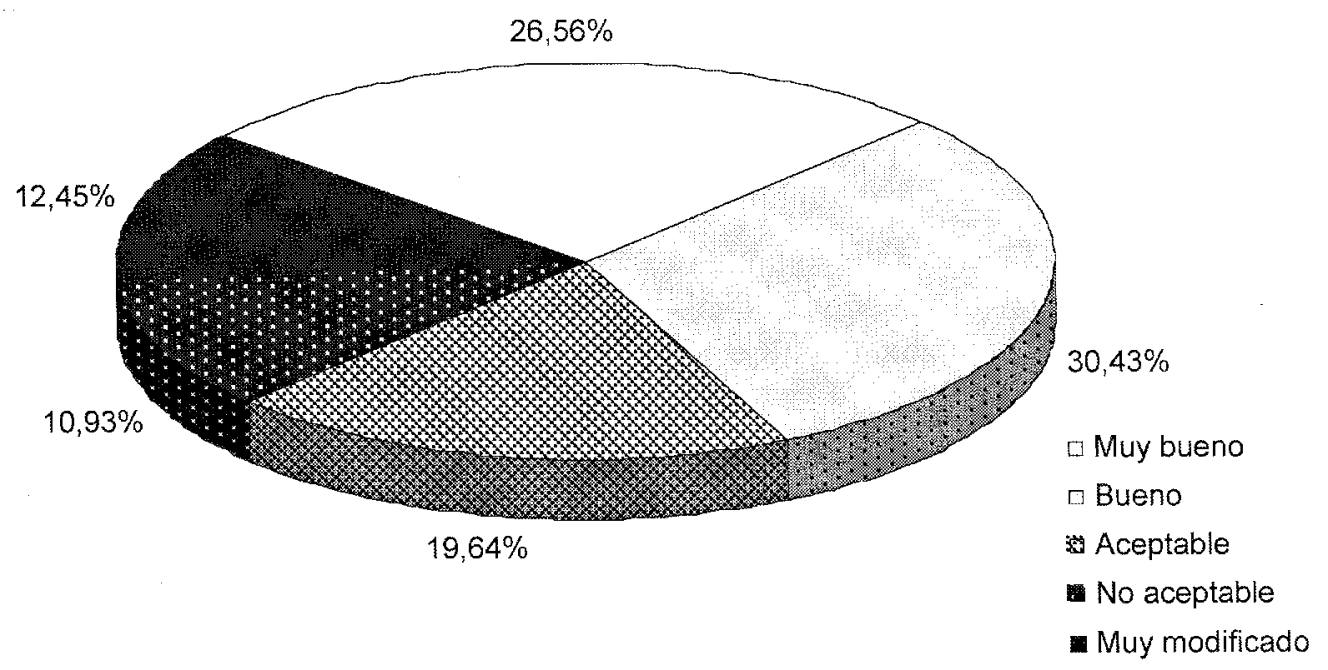

Figura 3. Estado de los indicadores hidromorfológicos en los ríos de Aragón: porcentajes por número de sectores funcionales.

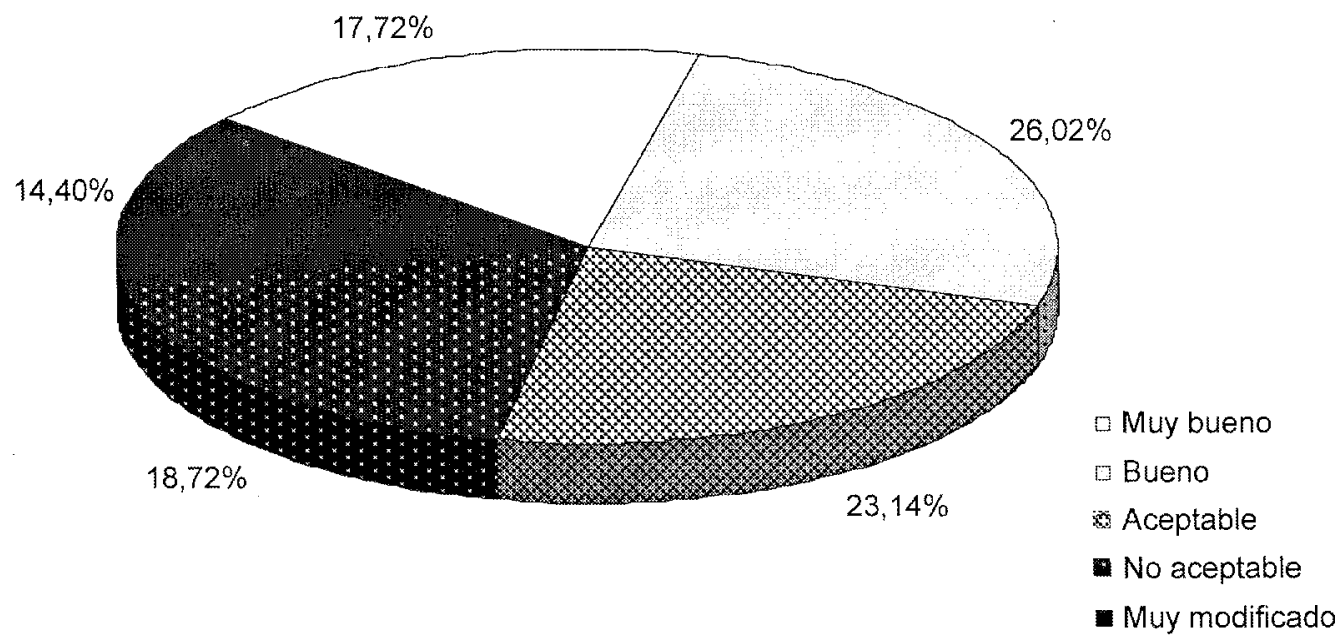

Figura 4. Estado de los indicadores hidromorfológicos en los ríos de Aragón: porcentajes por longitud de la red fluvial. 
b) Se observa que el valor máximo (100) es alcanzado por tan sólo 12 sectores funcionales: Ara 2, Aragón Subordán 2, Aragón Subordán 4, Aurín 1, Bellós 2, Bellós 3, Ésera 2, Ésera 3, Estarrún 1, Mascún 2, Osía 1 y Yaga 2. Es probable que si se hubiera podido hacer más trabajo de campo y por tanto puntuar con exactitud más sectores funcionales de los que se estiman como de estado muy bueno, algunos de ellos habrían alcanzado también el valor 100 .

c) Los valores más bajos corresponden a tramos embalsados y a sectores al pie de presas, así como a cursos bajos de cauces canalizados o muy constreñidos y con las riberas eliminadas.

Se observa en líneas generales una mayor calidad hidromorfológica en los cursos de montaña, siempre que no estén afectados por obras de regulación o derivaciones.

d) Pueden calificarse el Ara, el Isábena, los afluentes del Aragón por la derecha (en especial Estarrún, Subordán y Veral), el Guarga y algunos pequeños afluentes de los altos Ara, Cinca, Ribagorzana o Alcanadre (Arazas, Barrosa, Bellós, Yaga, Yesa, Irués, Baliera, Mascún, etc.) como los ríos de mayor calidad, ya que en sus recorridos completos su estado hidromorfológico se valora como muy bueno o bueno. El Aragón Subordán es el único río de aquellos que mantienen en todo su recorrido el buen estado que cuenta con dos tramos con valor máximo 100. También el Ésera tiene dos sectores que llegan a 100 puntos, pero aguas abajo se deteriora su situación.

e) La valoración global de los ríos de Aragón desde una perspectiva hidromorfológica es aceptable, lo cual no debe llevar a optimismo o conformismo. Son muchos los sectores funcionales que deberían mejorar su estado y requieren labores de restauración.

Las condiciones específicas de referencia de cada tipo de curso fluvial desde una perspectiva hidromorfológica responden siempre a los siguientes caracteres:

-No existen alteraciones antropogénicas de los valores de los indicadores de calidad hidromorfológicos o son de muy escasa importancia.

-El caudal, la hidrodinámica del sistema fluvial, la continuidad del mismo y las condiciones morfológicas corresponden totalmente o casi totalmente a las condiciones inalteradas.

-La complejidad, la funcionalidad y la dinámica del sistema fluvial son acordes con las características topográficas, hidroclimáticas y geomorfológicas del tipo.

El tramo de referencia de cada tipo es el que reúne las condiciones óptimas o está más próximo a las mismas, ya que en muchos tipos no se da en la actualidad en 
Aragón ningún tramo en muy buen estado ecológico desde indicadores hidromorfológicos (apartado 1.3 del Anexo II de la Directiva). En tales casos podría recurrirse a modelos, a situaciones óptimas a juicio de los expertos del equipo, a situaciones históricas (para lo cual puede compararse la situación actual con la observable en fotografías aéreas antiguas) e incluso a ejemplos de otros países o regiones, tal como recomienda el Guidance standard CEN TC 230/WG 2/TG 5: N32. Entendemos que es posible encontrar, o en otros momentos o en otros espacios geográficos, casos que alcancen el valor máximo 100 de nuestra escala, valor que marca las condiciones de referencia, no existiendo para ningún tipo (salvo para los embalses, que ya de por sí no son ríos) cortapisas no antrópicas que impidan alcanzar ese valor.

Los tramos que han obtenido la valoración más alta en cada uno de los tipos son los más próximos a las condiciones de referencia y se podrían considerar por tanto, a escala de Aragón, los modelos o ejemplos de referencia, aunque en muy pocos casos alcanzan el valor 100 .

Tabla 1. Modelos de referencia de cada tipo de curso fluvial.

\begin{tabular}{|c|c|c|c|}
\hline n. ${ }^{-}$ & código & tipo de curso fluvial & modelo de referencia \\
\hline 1 & A1.P.GI & de alta montaña pirenaica encajados & $\begin{array}{l}\text { Estarrún 1, Aurín 1, Osía } 1 \\
\text { y Bellós } 2 \text { (100 puntos) }\end{array}$ \\
\hline 2 & A1.P.G4 & de alta montaña pirenaica en valles en artesa & $\begin{array}{l}\text { Ara 2, Subordán } 2 \text {, Ésera } 2 \text {, } \\
\text { Ésera 3, Subordán } 4(100 \text { p. })\end{array}$ \\
\hline 3 & A1.I.G1 & de alta montaña ibérica encajados & Cabriel $2(94 \mathrm{p})$. \\
\hline 4 & A1.I.G2 & de alta montaña ibérica con ribera & - \\
\hline 5 & A2.P.G1 & de montaña media pirenaica encajados & Bellós 3, Yaga $2(100 \mathrm{p}$ ) \\
\hline 6 & A2.P.G2 & de montaña media pirenaica con ribera & Estarrún $2(95$ p.) \\
\hline 7 & A2,P.G3 & $\begin{array}{l}\text { de montaña media pirenaica con corredor ribereño } \\
\text { exenso }\end{array}$ & Ara $6(86 \mathrm{p})$ \\
\hline 8 & A2.I.G1 & de montaña media ibérica encajados & Aguas Vivas 3 (92 p.) \\
\hline 9 & A2.I.G2 & de montaña media ibérica con ribera & Pitarque $5(89 \mathrm{p})$. \\
\hline 10 & A3.P.G1 & medios y bajos pirenaicos encajados & Mascún $2(100$ p. $)$ \\
\hline 11 & A3.P.G2 & medios y bajos pirenaicos con ribera & Yaga $4(94 \mathrm{p})$ \\
\hline 12 & A3.P.G3 & $\begin{array}{l}\text { medios y bajos pirenaicos con corredor ribereño } \\
\text { extenso }\end{array}$ & 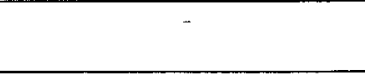 \\
\hline 13 & A3.I.G1 & medios y bajos ibéricos encajados & Isuela $3(92 \mathrm{p})$. \\
\hline 14 & A3.I.G2 & medios y bajos ibéricos con ribera & Deza $1(86 \mathrm{p})$. \\
\hline 15 & A3.I.G3 & medios y bajos ibéricos con corredor ribereño extenso & - \\
\hline 16 & G5.Q4 & ramblas & Ribota $3(85 \mathrm{p})$. \\
\hline 17 & G6-1 & embalses & - \\
\hline
\end{tabular}


En la selección de modelos de referencia se han seguido tres criterios:

-Se considera modelo de referencia de cada subtipo y de cada tipo el ejemplo de mayor valor del subtipo o tipo, pudiendo existir varios casos con la misma valoración.

-No cuentan con modelo de referencia aquellos subtipos que sólo aparecen en un caso o bien que no presentan ningún ejemplo o caso que haya obtenido la puntuación suficiente para ser considerado en estado "muy bueno".

-Tienen preferencia como modelos de referencia los casos para los que se ha podido realizar trabajo de campo, ya que en ellos la puntuación valorativa es real, mientras en los restantes es estimada.

En la tabla 1 se recogen los modelos de referencia de cada tipo en Aragón, siendo los tipos los derivados de la clasificación de Ollero et al. (2003). Puede observarse con claridad que sólo algunos sectores funcionales pirenaicos alcanzan el valor 100 . En tres tipos y 46 subtipos (más de la mitad) no hay ningún representante en Aragón que alcance los 85 puntos (muy buen estado). Son los cursos bajos y los cursos con ribera los que, muy afectados por actuaciones humanas, en pocos casos se aproximan a las condiciones de referencia.

\section{Agradecimientos}

El presente estudio fue parcialmente financiado por el Gobierno de Aragón en el contrato de investigación "Determinación del estado ecológico de los ríos de Aragón" (Universidad de Zaragoza-OTRI 2002-04/0375).

Todos los miembros del equipo quieren mostrar su gratitud y reconocimiento a Daniel Ballarín y Daniel Mora, auténticos autores principales de este trabajo, que han realizado más del 90\% de las observaciones y mediciones en los puntos de muestreo, siendo también los principales responsables del trabajo de gabinete y del control de todas las fichas e informaciones obtenidas.

\section{Referencias}

Bonada, N. et al. (2002) Intercalibración de la metodología GUADALMED. Selección de un protocolo de muestreo para la determinación del estado ecológico de los ríos mediterráneos. Limnetica, 21(34), 13-33.

Commission of the European Communities (2002) A guidance standard for assessing 
the bydromorpbological features of rivers. CEN TC 230/WG 2/TG 5 N32, 121.

Ladson, A.R. \& White, L.J. (1999) An Index of Stream Condition Reference Manual. Melbourne, Department of Natural Resources and Environment.

LAWA (2000) Gewässerstrukturgütebewertung in der Bundesrepublik Deutscbland, Verfabren für kleine und mittelgro_e Flie_gewässer. Berlin, Länderarbeitsgemeinschaft Wasser.

Munné, A., Solà, C. y Prat, N. (1998) QBR: un índice rápido para la evaluación de la calidad de los ecosistemas de ribera. Tecnología del agua, 175, 20-37.

Ollero, A., Echeverría, M.T., Sánchez Fabre, M., Auría, V., Ballarín, D. y Mora, D. (2003) Metodología para la tipificación hidromorfológica de los cursos fluviales de Aragón en aplicación de la Directiva Marco de Aguas (2000/60/CE). Geograpbicalia, 44, 7-25

Ollero, A., Ballarín, D., Díaz Bea, E., Echeverría, M.T., Montorio, R., Mora, D. y Sánchez Fabre, M. (2004) Reconocimiento inicial del estado ecológico de los ríos de Aragón mediante la caracterización de los indicadores bidromorfológicos del anexo $V$ de la Directiva 2000/60/CE. Departamento de
Medio Ambiente, Gobierno de Aragón (informe inédito).

Parsons, M., Thoms, M. \& Norris, R. (2002) Australian river assessment system review of pbysical river assessment metbods. A biological perspective. Monitoring River Health Initiative, Technical Report $n^{\circ} 21$, Commonwealth of Australia and University of Canberra.

Raven, P.J., Boon, P.J., Dawson, F.H. \& Ferguson, A.J.D. (1998) Towards an integrated aproach to classifying and evaluating rivers in UK. Aquatic Conservation. Marine and Fresbwater Ecosystems, 8(4), 383-393.

Siligardi, M. (Coord., 2003) I.F.F. Indice de funzionalità fluviale. Roma, Agencia Nazionale per la Protezione dell'Ambiente.

Tartar, P. (2001) Mise en oewore du système d'évaluation de la qualité pbysique des cours d'eau sur l'Andelle. Note de synthèse. DIREN Haute-Normandie.

Wallin, M., Wiederholm, T. \& Johnson, R.K. (2002) Guidance on establisbing reference conditions and ecological status class boundaries for inland surface waters. Water Framework Directive Common Implementation Strategy Working Group 2.3 REFCOND, CIS-WFD. 